DOI: $10.17725 /$ rensit.2019.11.357

\title{
Eliminating unauthorized access to the frequency resource of the geostationary satellite repeater
}

Andrey V. Sukhov

Moscow Aviation Institute, https:// mai.ru/

Moscow 125993, Russian Federation

Anton L. Tovbin

Informatics Research Center at the Ministry of Foreign Affairs of Russia, https://newnici. mid.ru/

Moscow 119200, Russian Federation

Vladislav V. Osipov

Scientific Research Institute for System Analysis of the Russian Academy of Sciences

Moscow 117218, Russian Federation

E-mail:subov@g-tl.ru,195555@list.ru,kt-mati@mail.ru

Received 28.08.2019, peer reviewed 16.09.2019, accepted 30.09.2019

Abstract. The technique of elimination of unauthorized use of the information transmission channel through the geostationary satellite repeater, based on the analysis of its information resource. The methodological approach is based on the application of differential entropy. The definition of information frequency resource is formulated, its properties are described. The method of calculating the a posteriori probability density of the coordinate vector of an unauthorized radio electronic means for the purpose of calculating the information frequency resource of the geostationary repeater satellite is presented.

Keyword: spectrum monitoring, information model, interference effect, electromagnetic compatibility, entropy.

UDC: 681.513 .6

Acknowledgments. This work was carried out as part of the state assignment of the Federal State Institution Scientific Center for Research and Development of the Russian Academy of Sciences (RAS) (basic research 14) on the topic No. 0065-2019-0001 "Software and tools for modeling, designing and developing elements of complex technical systems, software systems and telecommunication networks in various problem-oriented areas "(AAAA-A19-119011790077-1).

For citation: Andrey V. Sukhov, Anton L. Tovbin, Vladislav V. Osipov. Eliminating unauthorized access to the frequency resource of the geostationary satellite repeater. RENSIT, 2019, 11(3):357-368; DOI: 10.17725/rensit.2019.11.357.

\section{Contents}

1. INTRODUCTION (358)

2. MATHEMATICAL MODEL OF THE INFORMATION TRANSMISSION CHANNEL (358)

3. Estimation OF COORDINATE VECTOR OF AN UNAUTHORIZED TRANSMITTING STATION (359)
4. SATELLite INFORMATION FREQUENCY RESOURCE (361)

5. Optimal ESTIMATION OF THE STATE VECTOR OF AN UNAUTHORIZED ELECTRONIC STATION (363)

6. Conclusion (367)

REFERENCES (368) 


\section{INTRODUCTION}

Nowadays a lot of information is transmitting through geostationary satellite transponder (GST) because of the development of needs for the global informational exchange among multiply users. The using of this method of informational exchange is especially convenient when transferring information is over long distances, in conditions of sufficiently long sessions of information exchange and when sufficiently large amounts of information are transferring.

But such channels are not always used sanctioned. There are a lot of opportunities for the opening of the protection of informational exchange networks and the gaining access to the data channels (DC) because of the development of the computer technologies. The struggle against unauthorized using of DC with GST may be organized with the using of one or two GST and Earth control stations (ECS) associated withit. Thedetermination of coordinates of an unauthorized radio electronic station (URES) is made by using ECS, the unauthorized access session is logged and the application to the Radiocommunication Bureau of the International telecommunication community (ITC) is being prepared, on the basis of which administrative measures are taken to prevent the operation of the URES. Such a problem for the present situation to determine the coordinates of the transmitter by various methods devoted a lot of publications [ $1-4$, etc.].

This article discusses the method of the determining of the coordinates of the URES using one GST and one associated ECS. The scheme of the information exchange is shown in Fig. 1. This figure also shows the

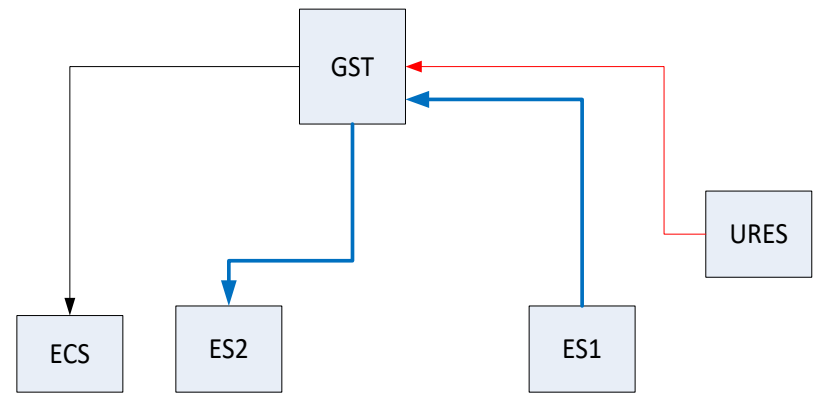

Fig. 1. The scheme of the information exchange through GST.

authorized DC between the first and second Earth stations (ES).

The URES transmitter uses the frequency resource of GST and essentially it is a source of interference. It is possible to determine its coordinates by a small Doppler shift of the received frequency, formed as a result of the constant movement of GST in some area of space relative to the position given for it in the geostationary orbit. Such shifts may be tens of kilometers [1] and the trajectory of the GST are known.

\section{MATHEMATICAL MODEL OF THE INFORMATION TRANSMISSION CHANNEL}

Consider the phenomenological model of the data channel, for which the important factors are the signal received at the input of the receiver of the Earth control station, the coordinates of the transmitter and the carrier frequency of the signal emitted by the transmitter.

According to [5] the observation equation has the form:

$z(t)=U(t) \cos \left(\omega_{T} t+\varphi(t)\right)+n(t)$,

where $U(t)$ - the signal amplitude; $n(t)$ - the channel additive white Gaussian noise with characteristics:

the mathematical expectation:

$\mathrm{M}(\mathrm{n}(\mathrm{t}))=0$,

the dispersion (second central moment): 


$$
M\left(n\left(t_{2}\right) n\left(t_{2}\right)\right)=\sigma_{n}^{2}=N_{0} / 2 \delta\left(t_{2}-t_{1}\right),
$$

$N_{0}$ - the one-way spectral noise power density, $\delta()-$ Delta function; the density of the channel noise:

$$
p(n)=\frac{1}{\sqrt{2 \pi} \sigma_{n}} e^{-\frac{n^{2}}{2 \sigma_{n}^{2}}},
$$

$\varphi(t)$ - the random phase shift, determined by the double Doppler frequency shift on the signal propagation paths transmitter URES GST and GST - Earth receiving station, and described in accordance with the models of parameters of radio signals presented in [6], the equation of state:

$$
\left\{\begin{array}{l}
\dot{\varphi}(t)=\omega(t), \\
\dot{\omega}(t)=-\alpha \omega(t)+n_{\omega}(t),
\end{array}\right.
$$

$n_{\omega}$ - the parametrical Gaussian noise, same described in (2) - (4) with the zero mathematical expectation and the dispersion (standard deviation) $\sigma_{\omega}^{2}$.

The results of the frequency measurements at the Earth control station is used for the estimation of the location of the unauthorized signal source. The frequency of the received signal $f_{R}$ is associated with the coordinates of the transmitting station $r_{T}$ in the form:

$$
f_{R}=\left[f_{T}\left(1+\frac{v_{s}^{t}\left(r_{T}-r_{s}\right)}{c\left\|r-r_{s}\right\|}\right)+\Delta_{f}\right]\left(1+\frac{v_{D}}{c}\right),
$$

where $f_{R}$ - the carrier frequency of the received signal; $f_{T}$ - the carrier frequency of the transmitting signal; $v_{S}$ - the velocity vector of the satellite in the observation period $v_{s}^{t}=\left(\dot{x}_{s}, \dot{y}_{s}, \dot{z}_{s}\right), \quad r_{s}-$ the position vector of the satellite in the observation period $r_{s}^{t}=\left(x_{s}, y_{s}, z_{s}\right), r_{T}$ - the position vector of the transmitter $r_{T}^{t}=\left(x_{T}, y_{T}, z_{T}\right), \Delta_{f}$ - the frequency offset in the satellite repeater; $v_{D}$ - the scalar rate of range change between satellite and receiver; $c$ - the spread speed of the signal, $\mathrm{c}$
$=3 \cdot 10^{8} \mathrm{M} / \mathrm{c}$; the upper index «t» denotes an algebraic transposition operation.

Cartesian geocentric coordinate system is used to determine vectors $r_{S}$ и $r_{T}$.

\section{ESTIMATION OF COORDINATE VECTOR OF THE UNAUTHORIZED TRANSMITTING STATION}

We will use the expression (6) to determine the coordinates of the unauthorized transmitter.

It is fair for frequency of the received signal with expression (1) and with that frequency is derivative on time from a phase of a signal:

$$
f_{R}=\frac{1}{2 \pi}\left(\omega_{T}+\dot{\varphi}(t)\right) \text {. }
$$

We will use the geocentric Cartesian coordinate system as the coordinate system. In this coordinate system, the difference between the position vectors of the GST and the transmitter has the following representation:

$r_{T}-r_{s}=\left(\begin{array}{c}x_{T}-x_{s} \\ y_{T}-y_{s} \\ z_{T}-z_{s}\end{array}\right)$.

The norm of the difference of these vectors has the following representation:

$$
\left\|r_{T}-r_{s}\right\|=\sqrt{\left(x_{T}-x_{s}\right)^{2}+\left(y_{T}-y_{s}\right)^{2}+\left(z_{T}-z_{s}\right)^{2}}
$$

In consideration of (7) - (9) expression (6) has the representation:

$$
\begin{aligned}
& \frac{1}{2 \pi}\left(\omega_{T}+\dot{\varphi}(t)\right)= \\
& =\left[f_{T}\left(1+\frac{\dot{x}_{s}\left(x_{T}-x_{s}\right)+\dot{y}_{s}\left(y_{T}-y_{s}\right)+\dot{z}_{s}\left(z_{T}-z_{s}\right.}{c \sqrt{\left(x_{T}-x_{s}\right)^{2}+\left(y_{T}-y_{s}\right)^{2}+\left(z_{T}-z_{s}\right)^{2}}}\right)+\Delta_{f}\right] \times \\
& \times\left(1+\frac{v_{D}}{c}\right) .
\end{aligned}
$$

Convert expression (10) to a more convenient representation. Enter the designation for this:

$B_{s}=1+v_{D} / c$ 
and we present the dependence of the measured parameter, which is a random component of the signal phase $\varphi(t)$, from the unknown parameters of the unauthorized RES - coordinates of the transmitter and the carrier frequency of the transmitter:

$$
\begin{aligned}
& \dot{\varphi}(t)= \\
& =B_{s} \omega_{T} \frac{\dot{x}_{s}\left(x_{T}-x_{s}\right)+\dot{y}_{s}\left(y_{T}-y_{s}\right)+\dot{z}_{s}\left(z_{T}-z_{s}\right)}{c \sqrt{\left(x_{T}-x_{s}\right)^{2}+\left(y_{T}-y_{s}\right)^{2}+\left(z_{T}-z_{s}\right)^{2}}}+ \\
& +\omega_{T}\left(B_{s}-1\right)+\Delta_{\omega} B_{s},
\end{aligned}
$$

where $\Delta_{\omega}=2 \pi \Delta_{f}, \omega_{T}=2 \pi f_{T}$.

In order to be able to estimate the parameters of the unauthorized RES, we will use the decomposition of the right part of the expression (11) into a Taylor series in the vicinity of the current estimate of the coordinates of the URES on the ground and the current estimate of the transmitter frequency and limit ourselves to members of the first order of smallness.

It is required to find the partial derivatives of the right part of the expression (11) on the vector of the estimated parameters $g(t)$ of the URES for this purpose. The vector of the estimated URES parameters consists of the following components: the coordinates of the URES on the ground and the carrier frequency of the transmitted signal:

$$
g^{*}(t)=\left(\begin{array}{c}
x_{T}^{*}(t) \\
y_{T}^{*}(t) \\
z_{T}^{*}(t) \\
\omega_{T}^{*}(t)
\end{array}\right) .
$$

The estimation of the parameters of the vector $g(t)$ will be carried out at a sufficiently long interval of observation time to ensure the required accuracy. So even if the components of this vector are stationary, the current estimates will change over time.

The partial derivatives of the function $\omega(g(t), t)=\dot{\varphi}(t)$ on the vector $g(t)$ on the basis of (11) have the following form:

$$
\begin{aligned}
& \Omega_{x}(t)=\frac{\partial \omega(g(t), t)}{\partial x_{T}}= \\
& =\frac{B_{s} \omega_{T} \dot{x}_{s}}{c \sqrt{\left(x_{T}-x_{s}\right)^{2}+\left(y_{T}-y_{s}\right)^{2}+\left(z_{T}-z_{s}\right)^{2}}}- \\
& -\frac{B_{s} \omega_{T}\left(x_{T}-x_{s}\right)}{c} \times \\
& \times \frac{\dot{x}_{s}\left(x_{T}-x_{s}\right)+\dot{y}_{s}\left(y_{T}-y_{s}\right)+\dot{z}_{s}\left(z_{T}-z_{s}\right)}{\left[\left(x_{T}-x_{s}\right)^{2}+\left(y_{T}-y_{s}\right)^{2}+\left(z_{T}-z_{s}\right)^{2}\right]^{3 / 2}}, \\
& \Omega_{y}(t)=\frac{\partial \omega(g(t), t)}{\partial y_{T}}=
\end{aligned}
$$$$
=\frac{B_{s} \omega_{T} \dot{y}_{s}}{c \sqrt{\left(x_{T}-x_{s}\right)^{2}+\left(y_{T}-y_{s}\right)^{2}+\left(z_{T}-z_{s}\right)^{2}}}-
$$$$
-\frac{B_{s} \omega_{T}\left(y_{T}-y_{s}\right)}{c} \times
$$

$\times \frac{\dot{x}_{s}\left(x_{T}-x_{s}\right)+\dot{y}_{s}\left(y_{T}-y_{s}\right)+\dot{z}_{s}\left(z_{T}-z_{s}\right)}{\left[\left(x_{T}-x_{s}\right)^{2}+\left(y_{T}-y_{s}\right)^{2}+\left(z_{T}-z_{s}\right)^{2}\right]^{3 / 2}}$,

$\Omega_{z}(t)=\frac{\partial \omega(g(t), t)}{\partial z_{T}}=$

$=\frac{B_{s} \omega_{T} \dot{z}_{s}}{c \sqrt{\left(x_{T}-x_{s}\right)^{2}+\left(y_{T}-y_{s}\right)^{2}+\left(z_{T}-z_{s}\right)^{2}}}-$

$$
-\frac{B_{s} \omega_{T}\left(z_{T}-z_{s}\right)}{c} \times
$$

$\times \frac{\dot{x}_{s}\left(x_{T}-x_{s}\right)+\dot{y}_{s}\left(y_{T}-y_{s}\right)+\dot{z}_{s}\left(z_{T}-z_{s}\right)}{\left[\left(x_{T}-x_{s}\right)^{2}+\left(y_{T}-y_{s}\right)^{2}+\left(z_{T}-z_{s}\right)^{2}\right]^{3 / 2}}$,

$\Omega_{f}(t)=\frac{\partial \omega(g(t), t)}{\partial \omega_{T}}=$

$=B_{s} \frac{\dot{x}_{s}\left(x_{T}-x_{s}\right)+\dot{y}_{s}\left(y_{T}-y_{s}\right)+\dot{z}_{s}\left(z_{T}-z_{s}\right)}{c \sqrt{\left(x_{T}-x_{s}\right)^{2}+\left(y_{T}-y_{s}\right)^{2}+\left(z_{T}-z_{s}\right)^{2}}}+$ $+\left(B_{s}-1\right)$.

We expand the function $\omega(g(t), t)=\dot{\varphi}(t)$ in the vicinity of some point $t_{k}$ into Taylor series [9]:

$$
\begin{aligned}
& \omega(g(t), t)=\dot{\varphi}(t) \approx \\
& \approx \Omega_{x}\left(t_{k}\right)\left(x_{T}(t)-x_{T}\left(t_{k}\right)\right)+ \\
& +\Omega_{y}\left(t_{k}\right)\left(y_{T}(t)-y_{T}\left(t_{k}\right)\right)+ \\
& +\Omega_{z}\left(t_{k}\right)\left(z_{T}(t)-z_{T}\left(t_{k}\right)\right)+ \\
& +\Omega_{f}\left(t_{k}\right)\left(f(t)-f\left(t_{k}\right)\right)+n_{\Omega}\left(t_{k}\right),
\end{aligned}
$$

where in the undefined parameter $n_{\Omega}\left(t_{k}\right)$ we take into account the residual term of 
the decomposition and a set of random factors with Gaussian behavior affecting the measurement results.

Given a sufficiently large number of such factors, as well as, considering the smallness of the remainder term of the series expansions, you can make the assumption about the Gaussian nature of the behavior parameter $n_{\Omega}\left(t_{k}\right)$. Then the distribution of the parameter $n_{\Omega}\left(t_{k}\right)$ will be described similarly to (2) - (4) with dispersion $\sigma_{\Omega}^{2}$.

Thus, when measuring the frequency of the signal from the URES transmitter quite a long time and a priori knowing the location of the GST at a given time, it is possible to filter the vector of unknown parameters of the URES. For this purpose, a system of equations (17) on a sufficient array of frequency measurements at different times $t_{k}$ should be used.

\section{SATELLITE INFORMATION FREQUENCY RESOURCE}

To determine the information space in which the information resource should be considered, we will use the differential entropy introduced by K. Shannon [7] and correctly defined by A.N. Kolmogorov [8], who called it differential entropy.

Differential entropy has been defined as a measure of uncertainty for continuous random variables. But it should be borne in mind that its absolute values are associated with the coordinate system in question, which does not allow one random variable entropy to make sufficiently reasonable judgments. At the same time, when comparing random variables, the use of the difference in their entropies avoids this drawback.
For a continuously distributed random variable $\mathrm{x}$ with a distribution density $\mathrm{p}(\mathrm{x})$, the differential entropy is defined as follows:

$$
H(x)=-\int_{-\infty}^{\infty} p(x) \log p(x) d x .
$$

For the concept of entropy, the base of the logarithm is not essential, because the base of the logarithm determines the units of information. For the base of the logarithm, two units will be bits, and for the natural logarithm, the units will be natas. It is more convenient to use natural logarithms, operations on which do not lead to the appearance of additional coefficients in mathematical transformations. Therefore, in the future, if no special reservations are made, it is assumed to use only natural logarithms.

In our case, the frequency resource of GST is considered. As the frequency parameter resource should take the distribution of the frequency of the received signal from URES. It is necessary to set as a measure some reference distribution for the URES resource for the correct application of differential entropy. This reference distribution is the distribution of the authorized operating frequency of the signal from the frequency range under consideration. For the operating frequency of the received authorized signal, the necessary statistical characteristics are a priori known, which makes it possible to determine the reference frequency distribution. Taking into account the impact on the transmission channel of information only Gaussian noise density distribution of the authorized frequency will also be subject to the normal distribution law:

$p\left(f_{r e f}\right)=\frac{1}{\sqrt{2 \pi} \sigma_{r e f}} e^{-\frac{\left(f_{r e f}-m_{r e f}\right)^{2}}{2 \sigma_{r e f}}}$,

where $m_{r f}$ - the mathematical expectation of the authorized frequency, $\sigma_{r f}-$ standard 
deviation of the values of the authorized frequency.

The value of its differential entropy for the normal distribution law (the differential entropy of the authorized frequency access) is known [7]:

$$
H\left(f_{r e f}\right)=\log \left(\sigma_{r e f} \sqrt{2 \pi e}\right),
$$

where $e$ - the basis of the natural logarithm.

We will use its entropy to determine the information frequency resource (IFR).

Definition: information frequency resource of the GST is the deviation of the differential entropy of the authorized frequency received by the Earth control station, due to interference from the URES.

Thus, the information frequency resource is presented in the form of:

$$
R_{i f r}=H\left(f_{\text {ref }}\right)-H\left(f_{\text {unref }}\right) \text {, }
$$

where $H\left(f_{\text {unrer }}\right)$ - differential entropy of unauthorized frequency of the received signal (differential entropy of unauthorized frequency access).

This definition of information frequency resource allows you to get the following important properties:

1. The information frequency resource is not bound to the differential entropy measurement scale. This property is determined by the presence of a difference term, which eliminates the dependence of the differential entropy on the coordinates.

2. In the absence of a signal from the URES, the value of the information frequency resource corresponds to the value of the differential entropy of the authorized access of GST, which characterizes the potential accuracy characteristics of GST, and that, in turn, can be used as a comparative indicator of GST.
3. The values of the information frequency resource due to its independence from the coordinates can be used as regulatory parameters at the conclusion of international agreements on the sharing of frequencies.

4. Tables and graphs of information frequency resource dynamics can be used in the preparation of universal calendar plans and recommendations to eliminate unauthorized access to the frequency resource.

In the whole statement of the problem, the information frequency resource should be maximized, and ideally, when operating of URES, it should be as close to zero as possible, which corresponds to the definition of the URES coordinate vector with the potential accuracy of the system with GST.

In the absence of unauthorized frequency access to the GST, the differential entropy of unauthorized access is zero due to the zero value of the density distribution of the frequency of unauthorized access in the entire area of its definition and the value of the information frequency resource is equal to:

$$
R_{i f r}=H\left(f_{r e f}\right) .
$$

The expression (17) shows the dependence of the unauthorized frequency of the received signal on the vector of coordinates of the URES. This expression is used to determine the location of the URES transmitter. And also on the basis of this expression it is possible to determine the differential entropy of the unauthorized frequency of the received signal.

Expression (17) is a linear function of its random parameters. Therefore, according to the properties of differential entropy, the value of the total differential entropy is also 
determined by the linear function of the entropy distributions of the random variables included in this function. In this case, we take into account the earlier conclusion about the normality of the laws of distributions of these random variables.

Based on the above, we obtain the following expression to determine the differential entropy of the unauthorized frequency:

$$
\begin{aligned}
& H\left(f_{\text {unref }}\right)=\Omega_{x}\left(t_{k}\right) H\left(x_{T}\right)+ \\
& +\Omega_{y}\left(t_{k}\right) H\left(y_{T}\right)+\Omega_{z}\left(t_{k}\right) H\left(z_{T}\right)+ \\
& +\Omega_{f}\left(t_{k}\right) H(f),
\end{aligned}
$$

where $H\left(x_{T}\right), H\left(y_{T}\right), H\left(z_{T}\right)-$ differential entropies for Cartesian coordinates of URES, $H(f)$ - the differential entropy of the frequency of the transmitter URES.

It should be noted that in the expression (23) there is no dependence on the parameters that determine the mathematical expectation of the laws of distribution of random variables, since they do not affect the differential entropy of the normal distribution law [7].

The technological cycle of the information monitoring process of the frequency resource consists of the following stages:

1. Monitoring of the Earth control station for authorized use of GST channels.

2. Detection of ground control station unauthorized use of GST in any frequency channel.

3. The inclusion of a ground control station of the optimal determinant of the coordinates of the URES and the optimal evaluation of the vector coordinates of the URES to obtain estimates with the required accuracy.
4. Carrying out organizational measures to disable the transmitter and prevent its further work.

5. Continuing of the monitoring of the ground control station for authorized use of GST channels.

\section{OPTIMAL ESTIMATION OF THE STATE VECTOR OF UNAUTHORIZED RADIOELECTRONIC WORKSTATIONS}

The estimation of the coordinate vector will be carried out in discrete time. Therefore, the transition from the equations of observation (1) and state (5) in continuous time should be made to the equations in finite differences. The observation equation for the $\mathrm{k}$ moment of time will take the form:

$$
Z_{k}=U_{0} \cos \left(\omega_{T k} t_{k}+\omega_{k}\right)+n_{k} .
$$

The differential equation of state in finite differences presents as follows:

$$
g_{k+1}^{f}=\Phi_{k+1} g_{k}^{f}+n_{f k+1} g_{k+1}^{f}=\Phi_{k+1} g_{k}^{f}+n_{f k+1} .
$$

In this case, taking into account the linearity of the equation of state (25), it is fair [6]:

$$
\Phi_{k+1}=\left(\begin{array}{cc}
1 & 1 / \alpha\left(1-e^{-\alpha \Delta t}\right) \\
0 & e^{-\alpha \Delta t}
\end{array}\right),
$$

where $\Delta t$ - sampling interval.

The expression for APD can be represented as follows [5]:

$$
p\left(g_{k+1} \mid\left\{z_{k+1}\right\}=\frac{p\left(z_{k+1} \mid g_{k+1}\right) p\left(g_{k+1} \mid\left\{z_{k}\right\}\right)}{p\left(z_{k+1} \mid\left\{z_{k}\right\}\right)},\right.
$$

where $\left\{z_{k}\right\}=z_{0}, z_{1}, z_{2}, \ldots, z_{k}$ - a set of implementations of the observed process.

As an approximate algorithm of optimal nonlinear filtering we will use the approximation of APD in the form of point masses on a rectangular lattice of indices [11-14]. We present APD as follows: 


$$
p\left(g_{k} \mid\left\{\mathrm{z}_{k}\right\}\right) \approx \sum_{\lambda_{k}} p\left(\lambda_{k}\right) \delta\left(g_{k}-\lambda_{k}\right) \Delta \lambda_{k},
$$

where $\Delta \lambda_{\mathrm{k}}=\Delta \lambda_{1} \Delta \lambda_{2} \ldots \Delta \lambda_{\mathrm{m}}, \Delta \lambda_{\mathrm{i}}, \Delta \lambda_{i}-$ the lattice spacing in the $i$ coordinate of the vector $g ; \delta\left(g_{\mathrm{k}}-\lambda_{\mathrm{k}}\right)-$ Delta function; $p\left(\lambda_{\mathrm{k}}\right)-$ function, satisfying the normalization condition:

$$
\sum_{\lambda_{k}} p\left(\lambda_{k}\right) \Delta \lambda_{k}=1, \forall k: p\left(\lambda_{k}\right) \geq 0 \text {. }
$$

Given the approximation (28), the expression (27) will take the form:

$$
\begin{aligned}
& p\left(g_{k} \mid\left\{z_{k}\right\}\right) \approx \\
& \approx \frac{p\left(z_{k} \mid g_{k}\right)}{C_{k}} \sum_{\lambda_{k}} p\left(\lambda_{k}\right) p\left(g_{k}, \lambda_{k}\right) \Delta \lambda_{k} .
\end{aligned}
$$

where

$$
\begin{aligned}
& p\left(g_{k}, \lambda_{k}\right)= \\
& =\int_{g_{k-1}} p\left(g_{k} \mid g_{k-1}\right) \delta\left(g_{k-1}-\lambda_{k}\right) d g_{k-1}=p\left(g_{k} \mid \lambda_{k}\right) .
\end{aligned}
$$

The normalizing constant $C_{k}$ is defined as follows:

$$
C_{\lambda k}=\sum_{\lambda_{k}} \sum_{g_{k}} p\left(\lambda_{k}\right) p\left(z_{k} \mid g_{k}^{\lambda}\right) p\left(g_{k}^{\lambda}, \lambda_{k}\right) \Delta \lambda_{k} \Delta g_{k}^{\lambda},
$$

where $g_{k}^{\lambda}$ - the values of the vector $g$ given on the set of lattice values $\lambda_{k}$.

The likelihood function $p\left(z_{k+1} \mid g_{k+1}\right)$, included in the expression (27) depends linearly only on random variables with a Gaussian distribution, so it also has a Gaussian distribution:

$$
\begin{aligned}
& p\left(z_{k+1} \mid g_{k+1}\right)= \\
& =\frac{1}{\sqrt{2 \pi} \sigma_{n}} \exp \left\{-\frac{1}{2 \sigma_{n}^{2}}\left(z_{k+1}-s\left(g_{k+1}^{\lambda}\right)^{2}\right\},\right.
\end{aligned}
$$

where $\sigma_{n}^{2}=N / 2 \Delta t$ - the variance of the channel noise.

The nodes of the grid shall conform to the following restrictions on the set of $T_{k}\left(\lambda_{k}\right)$ :

$$
T_{k}\left(\lambda_{k}\right)=\left\{\lambda_{k j} \in R^{M} \mid p\left(\lambda_{k}\right) \geq \delta_{0}\right\},
$$

where $\delta_{0}-$ a predetermined threshold level; $M$ - the dimension of the vector $g$.
The threshold level is determined by the noise intensity in the observation and state equations, and should be set to ensure stable operation of the filter. This practical recommendation is given by the authors [12].

The lattice nodes are given in the form of a uniform scale along the coordinates of the vector $g$ as follows:

$$
\begin{aligned}
& T_{k}\left(\lambda_{k}\right)= \\
& =\left\{\lambda_{k j} \in R^{M}|\forall j=1, \ldots, m:| \lambda_{j k} \mid<\mu \sigma_{\lambda}\right\},
\end{aligned}
$$

where $\mu$ - the value selected experimentally [12], its value is recommended to be set in the range from 1.5 to $3 ; \sigma_{\lambda}$ - standard deviation for the corresponding component of the coordinate vector.

Given (31), the joint density can be determined as follows:

$$
\begin{aligned}
& p\left(g_{k}, \lambda_{k}\right)= \\
& =\frac{1}{(2 \pi)^{M / 2}|D|} \exp \left\{-1 / 2\left(g_{k}-\lambda_{k}\right)^{t} D^{-1}\left(g_{k}-\lambda_{k}\right)\right\} .
\end{aligned}
$$

where superscript $t$ stands for transpose; $D$ - covariance matrix of the vector $g(t)$, which, taking into account the independence of the coordinates of the vector $g$ has a diagonal form:

$$
D=\left(\begin{array}{cccc}
\sigma_{x x}^{2} & 0 & 0 & 0 \\
0 & \sigma_{y y}^{2} & 0 & 0 \\
0 & 0 & \sigma_{z z}^{2} & 0 \\
0 & 0 & 0 & \sigma_{f f}^{2}
\end{array}\right) .
$$

Since it is assumed that the URES coordinates do not change in time and the vector of the coordinates being determined is subject only to Gaussian noise during the measurements, in accordance with [6] the equation of state in finite differences has the form:

$$
g_{k+1}=g_{k}+D_{\sigma} n_{g},
$$


where $n_{g}-$ parametric noise; $D_{\sigma}$ - matrix of standard deviations of parameters of URES vector:

$$
D_{\sigma}=\left(\begin{array}{cccc}
\left(\frac{\Delta t \sigma_{x x}^{2}}{2}\right)^{1 / 2} & 0 & 0 & 0 \\
0 & \left(\frac{\Delta t \sigma_{y y}^{2}}{2}\right)^{1 / 2} & 0 & 0 \\
0 & 0 & \left(\frac{\Delta t \sigma_{z z}^{2}}{2}\right)^{1 / 2} & 0 \\
0 & 0 & 0 & \left(\frac{\Delta t \sigma_{f f}^{2}}{2}\right)^{1 / 2}
\end{array}\right) .
$$

The covariance error matrix of vector estimates is defined as follows:

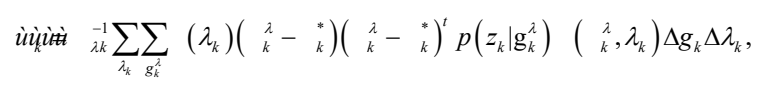

where $g_{k}^{*}$ - estimation of the coordinate vector at the $k$ step according to the selected Bayesian criterion of the average risk minimum, which can be obtained using the current approximation of the APD on a rectangular lattice of indices.

Based on the above expressions, the following algorithm of optimal nonlinear filtering is proposed (the block diagram of the filtering algorithm is shown in Fig. 2.)

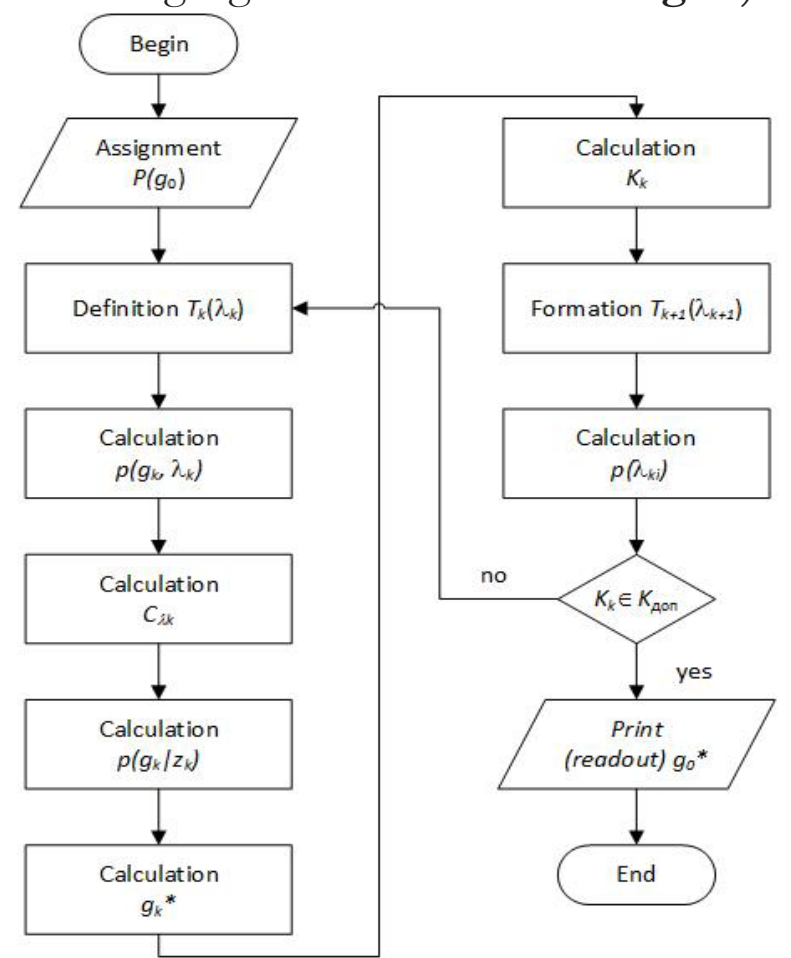

Fig. 2. The block diagram of the filtering algorithm of the URES coordinate vector.
1. At the first step of the filter operation using a priori information, truncated normal distribution laws are set for the coordinates of the unauthorized transmitter vector.

2. The nodes of the lattice $T_{k}\left(\lambda_{\mathrm{k}}\right)$ are determined in accordance with the expressions (34), (25) and the density of the distribution in the nodes is given by the a priori distribution.

3. The joint density $p\left(g_{k}, \lambda_{k}\right)$ is calculated based on the expression (36).

4. According to the expression (32) is calculated selection constant $C_{\lambda k}$.

5. APD $p\left(g_{k+1} \mid \tau_{k+1}\right)$ is calculated by expression (33).

6. Calculation of the current estimate of the vector of coordinates of $g_{k}^{*}$ according to selected functions of fines:

a. For a simple function of fines the coordinate with the maximum value of APD is determined.

b. For quadratic functions of fines is determined by the mathematical expectation for APD.

7. The covariance matrix of errors $K_{k}$ is calculated by expression (40).

8. In accordance with the obtained estimation of the coordinate vector, a lattice of indices is formed for the next step.

9. According to the expression distribution in the lattice $p\left(\lambda_{k i}\right)$ is calculated.

10. The calculations are repeated for items $2 \ldots 9$ before reaching the covariance matrix of the errors $K_{k}$ valid values for the precision of the coordinates. After that, the calculations are stopped and the results with the coordinates of the URES are transmitted to the coordinating bodies. 


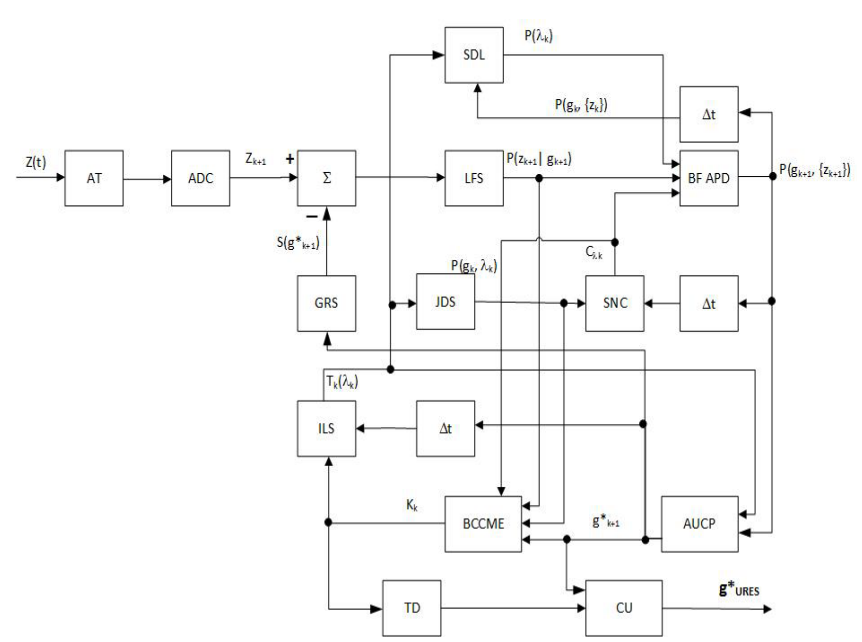

Fig. 3. The block scheme of the optimal digital filter.

Thus, an estimate of the URES coordinate vector can be obtained, but it is still necessary to estimate the information resource of GST in accordance with the recommendations given in section 2 .

The block diagram of the optimal digital filter with approximation of APD on a rectangular lattice of indices is shown in Fig. 3.

This diagram shows: AT - analog signal path that includes the antenna amplifier, preselector, amplifier high frequency and the possible local oscillator and intermediate frequency amplifier in the case of a too high carrier frequency signal; ADC analog-to-digital converter; $\Sigma$ - adder with one inverse and one direct input; LFS likelihood function shaper; SDL - shaper distribution in the lattice; $\Delta \mathrm{t}-$ delay element per quantization step to synchronize the operation of all elements of the scheme; BF APD - block of APD formation; GRS - generator of the reference signal based on the evaluation of the vector coordinates of the URES at the current step of the filter; JDS - joint density shaper; SNC - shaper of the normalizing constant; ILS - index lattice shaper; BCCME - block calculator the covariance matrix of the errors;

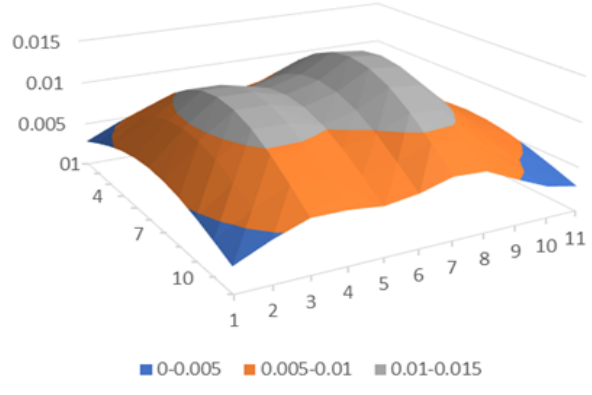

Fig. 4. The APD evolution. Initial filter operation mode.

AUCP - assessment unit of a continuous parameter; TD - a threshold device that is triggered when the errors in determining the coordinate vector of an acceptable level are reached; CU - crucial unit that issues coordinates URES the signal threshold of the device.

In the environment VisualStudio and using MSExcel spreadsheets, we performed a simulation of such a filter. The results of APD calculation for two coordinates on the plane are shown in Fig. 4 (initial filter operation mode), Fig. 5 (transient operation) and Fig. 6 (steadystate operation).

The dynamics of the information frequency resource of GST, obtained on the basis of APD processing, is presented in Fig. 7. Until the 10th cycle of the operation the URES did not include the transmitter. The value of the information frequency resource corresponds to the performance characteristics of the GST. At the 10th cycle of the filter operation the

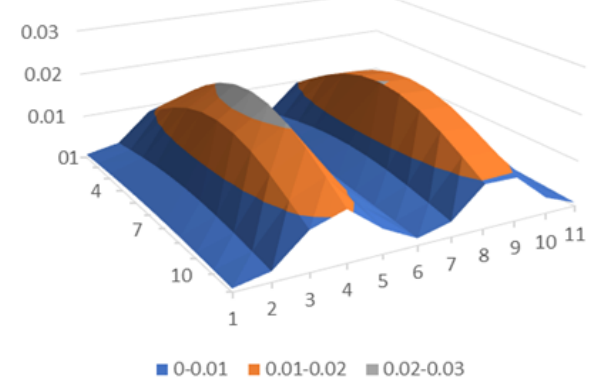

Fig. 5. The APD evolution. Transient operation. 
INFORMATION TECHNOLOGIES

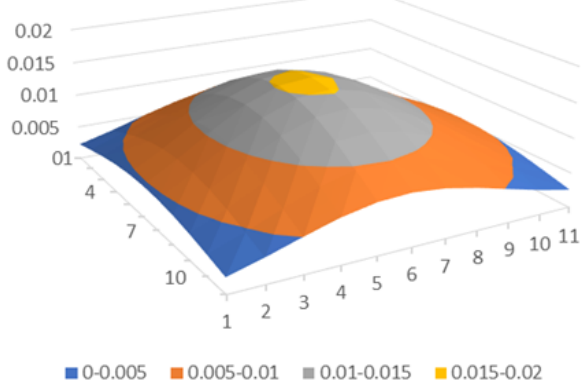

Fig. 6. The APD evolution. Steady-state operation.

activation of the transmitter URES passed and this event corresponded to the drop in the value of the information frequency resource. Further, up to the 52nd cycle of operation, the processing of the observed implementation of the input signal on the ECS was carried out. At the same time information frequency resource increased with increasing accuracy of estimation of the vector of coordinates of GST. Since the 52-th cycle the required accuracy of the vector coordinates URES was obtained and the shutdown of the transmitter URES occurred. The value of the information frequency resource returned to its original state.

The algorithms, obtained in the present work, designed for finding the information of the GST damage by function of the URES.

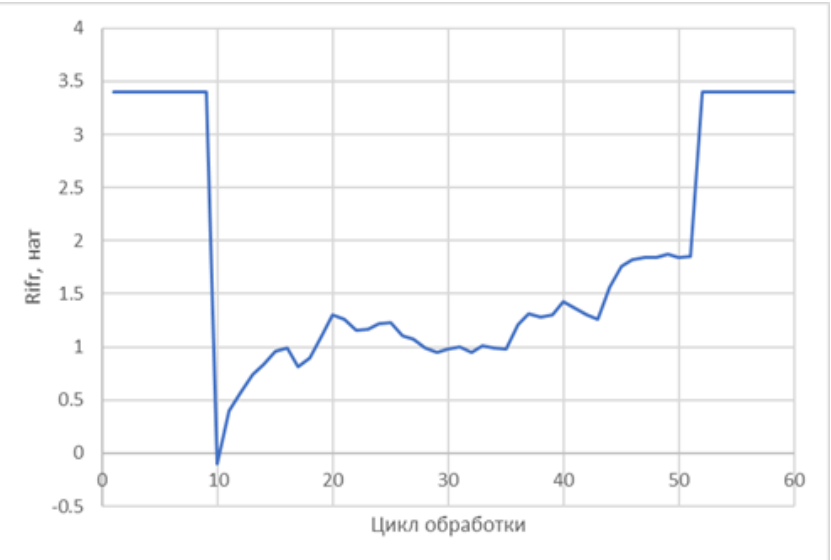

Fig. 7. Dynamics of information of the frequency resource.
ELIMINATING UNAUTHORIZED ACCESS TO THE $36 \mathbf{7}$ FREQUENCY RESOURCE OF THE GEOSTATIONARY...

\section{CONCLUSION}

Thus, the article considers the following provisions:

1. The phenomenological mathematical model of the CRPD is synthesized, comprising the GST, ECS, and URES, and defined the parameters needed for analysis and modeling of CRPD during the optimal estimation of the vector coordinates URES.

2. The partial differential decomposition is obtained by further transformation of the frequency tracking measurement equation. This decomposition allowed us to associate the unknown components of the vector of coordinates URES with the values of the measurements to allow for optimal estimation of the vector of coordinates with the use of optimal filtering algorithms.

3. The definition of information frequency resource based on differential entropy is formulated. The properties of IFR, defining a wide range of possibilities for its application, are described. IFR can be used as an indicator of the accuracy characteristics of GST. It can also be used as an indicator in international agreements on frequency sharing. Tables and graphs of information frequency resource dynamics can be used in the preparation of universal calendar plans and recommendations to eliminate unauthorized access to the frequency resource.

4. Solved the problem of estimating the vector of coordinates URES if you use the same earth test monitoring station with the use of APD approximation in the form of a point-mass on a rectangular lattice of indices.

5. The stages of the technological cycle of the information monitoring process of the frequency resource are given and the modeling of the optimal receiver is carried out. The obtained dynamic values of the 
information of the frequency resource in different stages of operation of the GST.

\section{ACKNOWLedgments}

Work is made as part of national assignment for SRISA RAS (fundamental scientific research GP 14) on the topic No.0065-2019-0001 (AAAA-A19-119011790077-1).

\section{REFERENCES}

1. Handbook on Spectrum Monitoring. ITU, Switzerland, Geneva, 2011.

2. Chernyak VS. Multiposition radiolocation. Moscow, Radio i svyaz' Publ., 1993, 416 p.

3. Koets MA, Bentley RT. Satellite Based Geolocation Using a Single Geosynchronous Satellite and an Inverse Doppler Technique. SRI Publ., USA, S.A., 1999.

4. Reshetnikov VN, Savilkin S B, Sukhov AV. Monitoring chastotnogo resursa geostatsinarbykh sputnikovretranslyatorov $\mathrm{s}$ ispolzovaniem entropii pokrytiya [Monitoring frequency resource of the geostationary transponders using entropy cover]. Programmnye produkety $i$ sistemy, 2017, 1:119-123 (in Russ.).

5. Tikhonov VI. Optimalny priem signalov [Optimal signal reception]. Moscow, Radio i svyaz' Publ., 1983, 320 pp.

6. Tikhonor VI. Nelineynoe preobrazovanie sluchaynykh protsessov [Non-linear transformation of random processes]. Moscow, Radio i svyaz' Publ., 1986. 296 p.

7. Shannon K. Raboty po teorii informatsii $i$ kibernetike [Works on information theory and cybernetics]. Moscow, Inlit Publ., 1963, $830 \mathrm{p}$.

8. Kolmogorov AN. Teoriya informatsii $i$ teoriya algoritmov [Information theory and algorithm theory]. Moscow, Nauka Publ., 1987, 304 pp.
9. Korn G, Korn T. Spravochnik, po matematike [Handbook of mathematics]. Moscow, Nauka Publ., 1977, 831 p.

10. Sage A, Mels J. Teoriya otsenivaniya $i$ ee primenenie $v$ svyazi $i$ upravlenii [Evaluation theory and its application in communication and management]. Moscow, Svyaz' Publ., 1976, 496 p.

11. Loginov VP, Ustinov ND. Priblizhennye algoritmy nelineynoy filtratsii [Approximate nonlinear filtering algorithms]. Zarubez̧hnaya radioelektronika, 1975. 3:3-28.

12. Sukhov AV, Zaitsev MA. Modelnoalgoritmicheskoe obespechenie infomatsionnykh sistem upravleniya [Model-algorithmic support of control information systems: monograph]. Moscow, Witte Mos. Univ. Publ., 2016, 128 pp.

13. Bucy RS, Senne KD. Digital Synthessis of Nonlinear Filters. Automatica, 1971, 7:287-298.

14. Bucy RS. Bayes Theorem and digital Realisation for nonlinear Filters. Journ. Am. Astronaut. Soc., 1969, 17:80-94. 